\title{
Intrasubject repeatability of in vivo intervertebral motion parameters using quantitative fluoroscopy
}

\author{
Alexander Breen ${ }^{1} \cdot$ Rebecca Hemming ${ }^{2} \cdot$ Fiona Mellor $^{1} \cdot$ Alan Breen $^{3}$ D
}

Received: 6 August 2018 / Accepted: 30 November 2018 / Published online: 8 December 2018

(c) The Author(s) 2018

\begin{abstract}
Purpose In vivo quantification of intervertebral motion through imaging has progressed to a point where biomarkers for low back pain are emerging. This makes possible deeper study of the condition's biometrics. However, the measurement of change over time involves error. The purpose of this prospective investigation is to determine the intrasubject repeatability of six in vivo intervertebral motion parameters using quantitative fluoroscopy.

Methods Intrasubject reliability (ICC) and minimal detectable change (MDC) of baseline to 6-week follow-up measurements were calculated for six lumbar spine intervertebral motion parameters in 109 healthy volunteers. A standardised quantitative fluoroscopy $(\mathrm{QF})$ protocol was used to provide measurements in the coronal and sagittal planes using both passive recumbent and active weight-bearing motion. Parameters were: intervertebral range of motion (IV-RoM), laxity, motion sharing inequality (MSI), motion sharing variability (MSV), flexion translation and anterior disc height change during flexion.

Results The best overall intrasubject reliability (ICC) and agreement (MDC) were for disc height (ICC 0.89, MDC 43\%) and IV-RoM (ICC 0.96, MDC 60\%), and the worst for MSV (ICC 0.04, MDC 408\%). Laxity, MSI and translation had acceptable reliability (most ICCs $>0.60$ ), but not agreement (MDC $>85 \%$ ).

Conclusion Disc height and IV-RoM measurement using QF could be considered for randomised trials, while laxity, MSI and translation could be considered for moderators, correlates or mediators of patient-reported outcomes. MSV had both poor reliability and agreement over 6 weeks.
\end{abstract}

Graphical abstract These slides can be retrieved under Electronic Supplementary Material.
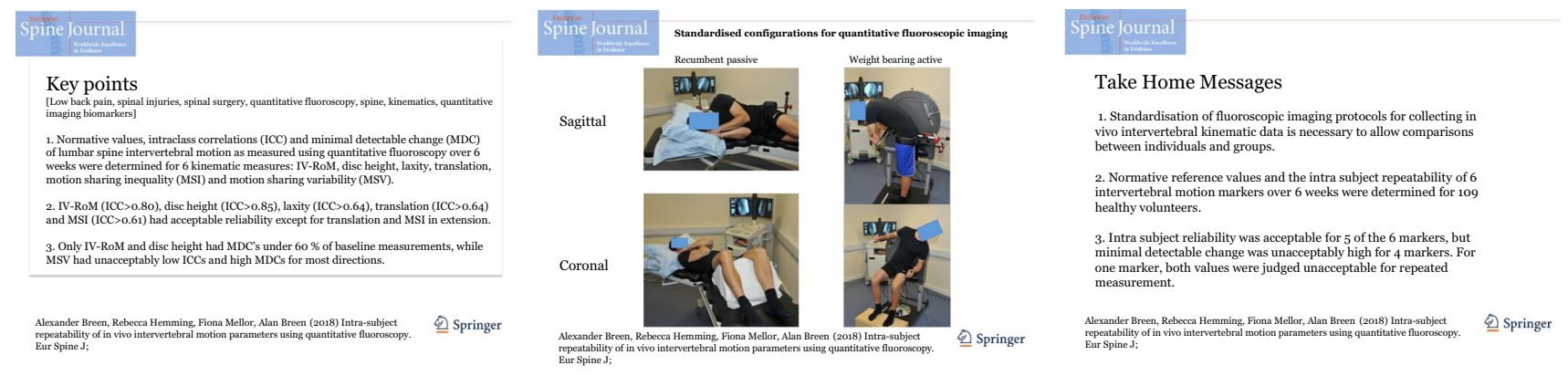

Keywords Low back pain $\cdot$ Spinal surgery $\cdot$ Kinematics $\cdot$ Quantitative imaging biomarkers

Electronic supplementary material The online version of this article (https://doi.org/10.1007/s00586-018-5849-9) contains supplementary material, which is available to authorized users.

Alan Breen

abreen4@bournemouth.ac.uk

Extended author information available on the last page of the article

\section{Background}

Low back pain is the world's largest cause of years lost to disability, but it usually has no objective diagnosis or known mechanism [1, 2]. Aberrant intervertebral motion in the lumbar spine as measured in vivo using standardised quantitative 
fluoroscopic imaging protocols $(\mathrm{QF})$ has been linked to nonspecific low back pain (NSLBP) as a biomarker [3-5], and many interventions exist to influence it [6-8]. This holds out the prospect of applying lumbar motion parameters as personalised biomarkers for the diagnosis of otherwise 'nonspecific' low back pain.

By improving understanding of mechanisms in individual patients, measurement of quantitative imaging biomarkers for back pain that takes advantage of such technologies could accelerate the development of new management approaches and facilitate more personalised care that may help avoid chronicity and/or resort-to-opioid medications [9]. However, quantitative imaging biomarkers are an emerging science [10], and measuring changes in motion parameters will always involve some error, either because of natural variation in the subject, variation in the measurement process or both [11]. Recommendations for scientific studies and regulatory submissions highlight the requirement to measure change; therefore, it is necessary to establish intrasubject repeatability over a credible intervention period for each parameter [10].

The dynamic measurement of continuous intervertebral motion in vivo is a relatively recent development, and intrasubject variation tests have tended to be limited to regional lumbar range of motion over short periods [12]. This has tended to confine the objective dynamic measurement of intervertebral function to cadaveric studies and computer models [13-17] providing little insight into individual living patients and representing a predicament in spine biomechanics research that has led to calls for in vivo, dynamic measurement methods of the multi-segmental spine and their validation. The hope is to make possible the production of individualised and, if possible, predictive models of functional spinal derangements $[18,19]$.

To provide such methods and allow them to be used to make valid comparisons between individuals, settings, populations and time points, two-dimensional (QF) systems have been developed that use standardised patient motion protocols to acquire multi-segmental, continuous image sequences from which intervertebral movement can be analysed with minimal behavioural variation. The resulting studies have provided early evidence that excessive intervertebral sagittal plane translation [20, 21], laxity [4], motion sharing inequality (MSI) [5], motion sharing variability (MSV) [22] and instant centres of rotation (ICRs) [23] are in various ways associated with spinal pain. Accuracy and observer repeatability studies have tended to support these parameters, as well as intervertebral range of angular motion (IV-RoM) and anterior disc height [24-26]. However, intrasubject repeatability data are lacking.

The intrasubject repeatability of intervertebral kinematic measures is also important when trying to decide whether a given parameter can be used in follow-up studies. This is typically expressed as the minimal detectable change (MDC), or measurement error, which is the change required to exceed the inherent variability in a truly unchanged population [11]. It reflects the smallest within-person variation, or change in score that can be interpreted as real and statistically significant, making it possible to decide in advance whether the degree of change that is of clinical interest can be detected with the technology at hand. This is different to the need to distinguish between subjects, when reliability measures, such as intraclass correlations, are preferred [27].

Aim of study The above parameters can be extrapolated from continuous multilevel intervertebral motion studies using QF. The aim of this study was to determine the intrasubject reliability (ICC) [28] and minimal detectable change $\left(\mathrm{MDC}_{95}\right)$ [11] of the repeated measurement of kinematic parameters during standardised active weight bearing and recumbent passive lumbar spine motion in flexion, extension, left- and right-side bending from L2-S1 using 2D quantitative fluoroscopy $(\mathrm{QF})$ in healthy individuals over a period of 6 weeks.

\section{Methods}

\section{Variables under consideration}

Intervertebral range of angular motion (IV-RoM) IV-RoM as measured with QF is the maximum angular rotation of intervertebral motion reached during bending (Fig. 1). In various forms, it is a very common biomechanical measure [29-31]. QF has been reported as measuring IV-RoM in the cervical spine with levels of interobserver agreement ranging from $0.3^{\circ}$ to $1.0^{\circ}$ (SEM) and reliability of $0.92-0.99$ (ICCs) [32] and in the lumbar spine with between $0.23^{\circ}$ and $0.76^{\circ}$ (SEM) and reliability of 0.94-0.99 (ICCs) [33].

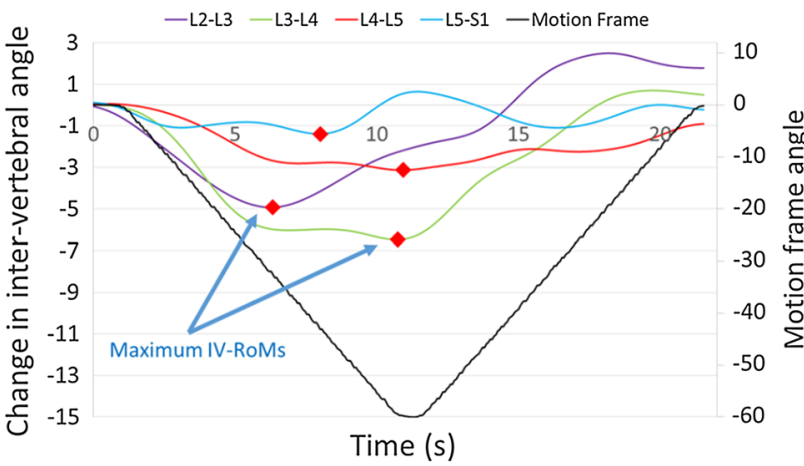

Fig. 1 Example of the identification of maximum intervertebral rotational range (IV-RoM) using a standardised lumbar left bending and return QF imaging of L2-S1. Note that the maximum IV-RoM does not necessarily occur at the maximum of motion frame range 
Sagittal translation Translation can be calculated for the sagittal plane in vertebral body units (VBU) which are converted to millimetres for presentation by multiplying the result by 35 , being the standard chosen for vertebral body depth in millimetres [34]. Intra- and interobserver agreement for translation using QF has been found to be $1.1 \mathrm{~mm}$ or less (SEM) with fair-to-substantial reliability (ICCintra 0.53-0.99, ICCinter 0.57-0.93) [35].

Laxity Laxity is a kinematic measure that reflects midrange intervertebral restraint in response to external forces [36]. It is used as a surrogate indicator of dynamic neutral zone length in in vivo studies and is also sometimes known as the initial attainment rate of intervertebral rotational displacement [37]. High values are evidence of disco-ligamentous microstrain or sub-failure and therefore a potential source of nociceptive pain [38]. Laxity is measured as the gradient of intervertebral motion in the initial $10^{\circ}$ of global motion from the mid-range position [39] (Fig. 2). The higher the ratio, the less the restraint within the vertebral linkage [40]. Reliability for laxity has been found to range from ICCintra 0.84-0.98 and ICCinter 0.92-0.98 [33].

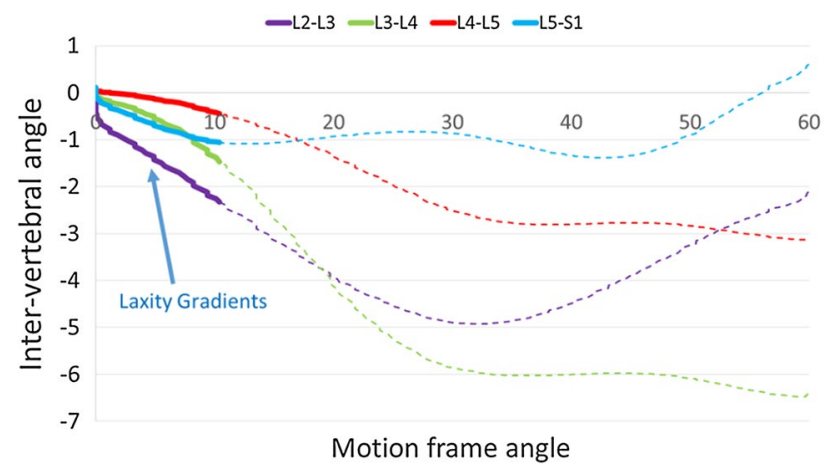

Fig. 2 Example of laxity (initial attainment rate) as initial gradients for four intervertebral levels
Anterior disc height Disc height is defined as the sum of the perpendicular distances of the anterior-inferior corner of the cranial vertebra and the anterior-superior corner of the caudal vertebra from the bisectrix between the two vertebral body mid-planes [34] (Fig. 3). Disc height is used to measure the effects of disc degeneration and end plate subsidence in relation to disc prostheses [41]. Anterior disc height, like translation, is also calculated in VBU for flexion and extension and subsequently converted to millimetres. It is calculated as a maximum for extension and a minimum for flexion. Reliability for disc height change for extension has been reported as ICCintra 0.65-0.97 and ICCinter 0.49-0.0.97, and for flexion as ICCintra 0.24-0.88 and ICCinter 0.64-0.99 [25].

Motion sharing inequality (MSI) and motion sharing variability (MSV) Asynchronous intervertebral motion during standardised trunk bending has been found to be greater in patients with nonspecific back pain than in controls and may represent a form of movement impairment [5, 22, 42]. Numerically, MSI is the average range of differences in the sharing of motion by each intervertebral level at each data point throughout the motion and reflects inequality of restraint across levels. MSV is calculated as the square root of the variance (or SD) of these differences throughout the motion. Both variables are derived from continuous proportional angular motion data (Fig. 4), and MSV may be considered to reflect intervertebral motion control. Details of these variables and methodologies have been published elsewhere $[5,42]$. However, no observer repeatability statistics have yet been published for MSI and MSV.

Instant centre of rotation (ICR) The ICR is conventionally the fulcrum of the arc of rotation of a vertebra with respect to its subjacent neighbour over a predetermined range. Its importance lies in the belief that it represents the centre of reaction force during loaded bending [43]. The more caudal its position, the more translation has accompanied the bend
Fig. 3 Measurement of anterior disc height in the a neutral and b flexed positions based on the sagittal mid-planes of adjacent vertebrae From Frobin et al. [34]
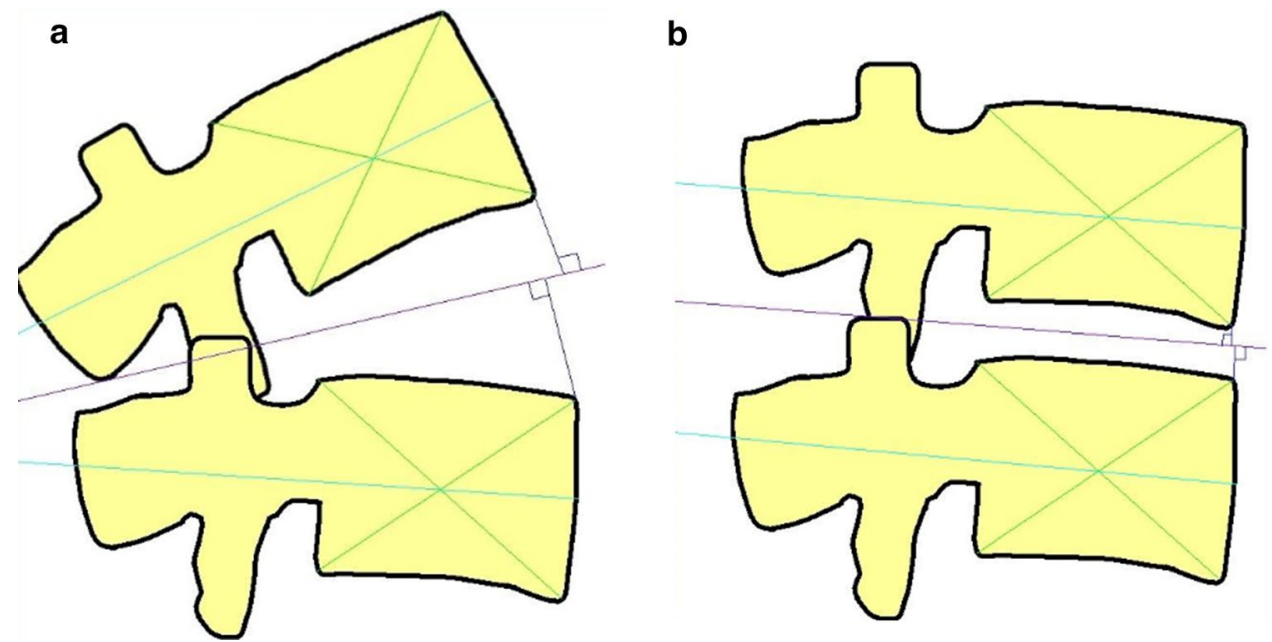


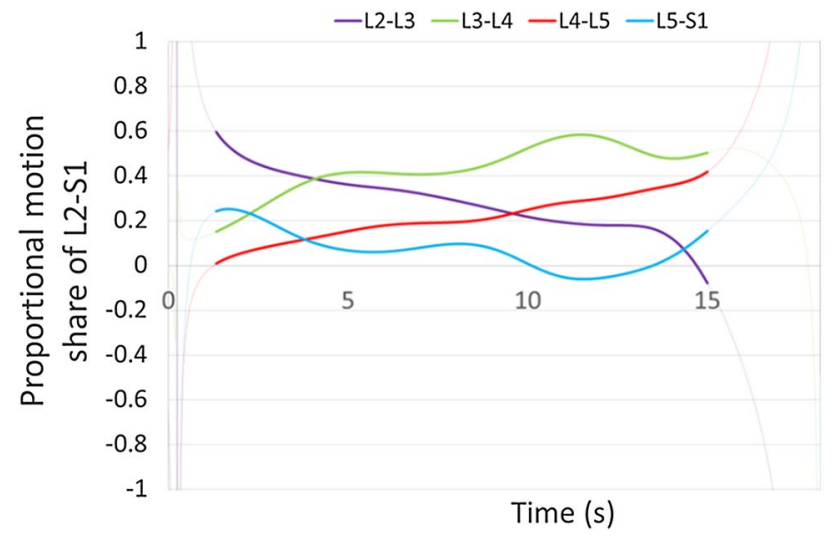

Fig. 4 Example of intervertebral proportional motion sharing at four intervertebral levels during outward and return motion. Motion sharing inequality (MSI) is calculated as the average of the maximum distances between levels at all data points and motion sharing variability (MSV) as the square root of their variance

over the chosen range. Unfortunately, it is prone to large errors for small rotations, making it difficult to gather large amounts of change data over time. However, for rotations greater than $5^{\circ}$, QF has substantial-to-excellent reliability (ICCintra 0.63-0.99 and ICCinter 0.62-0.88) [26].

Sample size calculation Sample size was calculated as the smallest number that would allow an assessment of intrasubject repeatability based on recognising a minimal change of $25 \%$ of the mean value for each kinematic index [11]. This allows an evaluation of the method to detect changes that are well within the upper reference limits found in previous studies. The width of the $95 \%$ confidence interval for the population within-subject standard deviation is given by:

$$
1.96 \frac{S_{\mathrm{w}}}{\sqrt{2 n(m-1)}}
$$

where $S_{\mathrm{w}}$ is the precision that can be estimated, $\mathrm{m}$ is the number of observations per subject and $n$ is the number of subjects required.

We wished to estimate to a precision of $1.96 \mathrm{SD}$ with two observations per subject and a confidence interval $\leq 0.25$ of the mean value of each parameter in healthy controls. Solving for $\mathrm{n}$ in the equation below returns $n=30.73$.

$\frac{1.96}{\sqrt{2 n(2-1)}}=0.25$

With 31 pairs of observations, according to central limit theorem, the sampling distribution of the mean will also approach a normal distribution, which will allow calculation of the baseline standard deviation for future power calculations. Therefore, to enable 31 participants to be imaged in each of the coronal and sagittal planes (to minimise radiation dosage to participants), upwards of 62 participants were needed. However, it was planned to recruit 150 participants with these inclusion criteria for a normative database, which is still in progress. Therefore, this target was exceeded.

Participant recruitment A convenience sample of 109 healthy control volunteers was recruited from staff, students and visitors of the AECC University College (Bournemouth, UK). Participants were included if they were aged 21-80, $\mathrm{BMI}<30$, with no history of previous back or abdominal surgery or spondylolisthesis, no medical radiation exposure of $>8 \mathrm{mSv}$ in the previous 2 years and no current pregnancy. Participants also had to have been free of any back pain that limited their normal activity for more than 1 day in the previous year. In order to restrict radiation dosage, within-subject measurements over 6 weeks were only carried out twice. Fifty-four received passive recumbent and active controlled weight-bearing QF investigations to the left and right (coronal plane), and 55 received passive recumbent and active weight-bearing controlled flexion and extension (sagittal plane) investigations of their lumbar spine motion. All participants had these procedures repeated 6 weeks later by the same operators using the same equipment at approximately the same time of the day. Informed consent was obtained from all participants, and ethical approval was obtained from the National Research Ethics Service (South West 3, 10/H0106/65).

Data collection The QF image acquisition and analysis procedures are further detailed in previous studies [5, 21, 22] (Fig. 5a-d). However, in order to minimise radiation dose, participants were allocated to either coronal or sagittal plane sequences.

All participants had both recumbent and weight-bearing imaging. For recumbent QF, participants lay on a movable table in which the trunk section was motorised and driven by a controller (Atlas Clinical Ltd.). This produced a bending angle of $40^{\circ}$ during separate left and right (coronal plane, subject supine) and flexion and extension (sagittal plane, subject-side lying) motion sequences during fluoroscopic screening. For active controlled weight bearing, participants sat on a stool with their backs against an upright motion frame fitted with arm rests which guided them through $40^{\circ}$ of left- and right-side bending. Participants receiving sagittal plane investigations stood with their right side against the motion frame with their pelvis secured and upper limbs supported on a projecting rest which guided them through $60^{\circ}$ of flexion angle (and return) using the same controller apparatus as for the recumbent procedure. The motion controllers accelerated at $6^{\circ} \mathrm{s}^{-2}$ for the first and second followed by a uniform $6^{\circ} \mathrm{s}^{-1}$ thereafter. The images were collected as single (not repeated) motion sequences at $15 \mathrm{~Hz}$ using a Siemens ARCADIS Avantic digital C-arm fluoroscope (Siemens GMBH) giving approximately 230 frames per sequence. All images were exported to a computer 


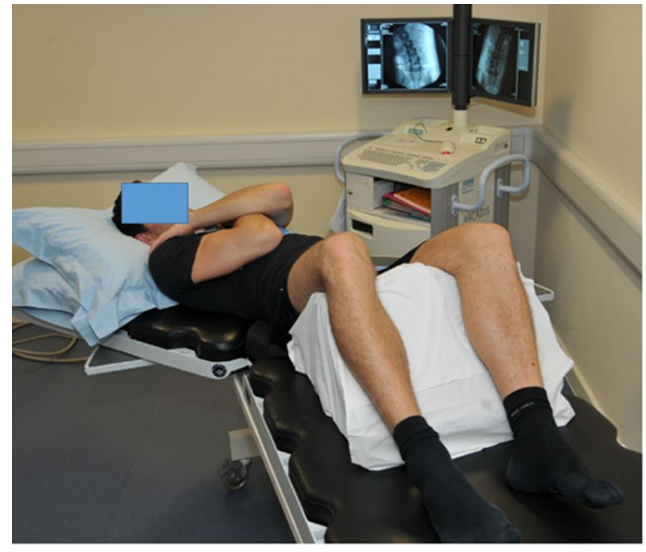

a

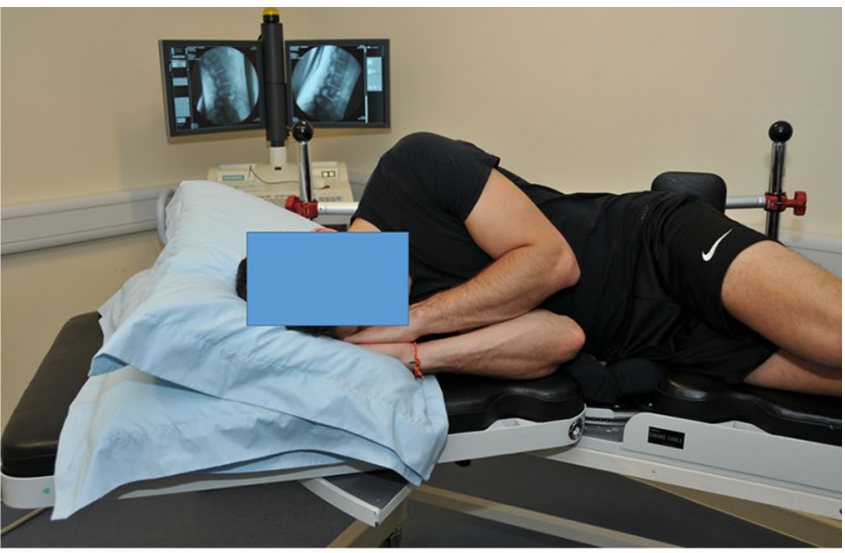

b

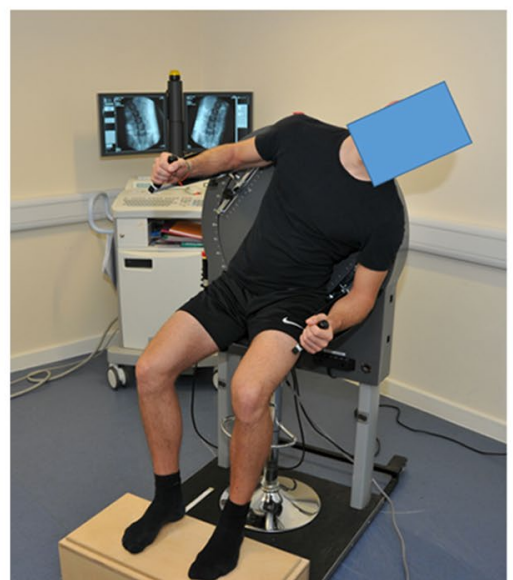

C

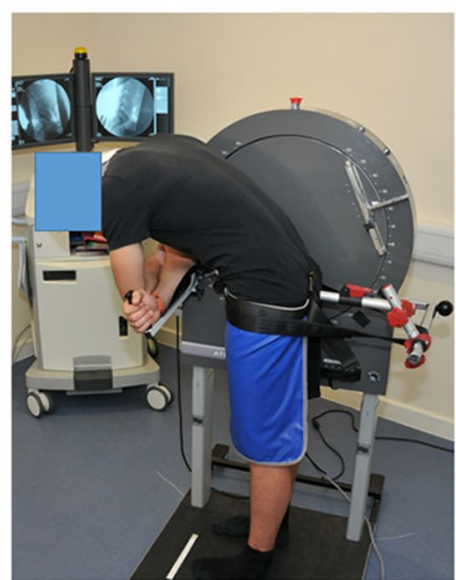

d

Fig. 5 a-d Positioning of participants for $\mathbf{a}$ passive recumbent coronal and $\mathbf{b}$ passive recumbent sagittal recumbent and $\mathbf{c}$ active weight-bearing coronal and $\mathbf{d}$ active weight-bearing sagittal imaging

workstation and analysed using manual first image registration and thereafter bespoke frame-to-frame tracking using codes written in MATLAB (v2011a-The MathWorks Inc.

\section{Calculation of kinematic parameters}

Maximum intervertebral rotation (IV-RoM), maximum sagittal translation in flexion, sagittal disc height during flexion (maximal in neutral to minimal in flexion), laxity (gradient of segment to trunk motion in first $10^{\circ}$ ), MSI (average proportional range shared between segments) and MSV (square root of the variance of the proportional range shared between segments) were calculated. Individual-level intervertebral motion data for each orientation (upright or lying) and direction (left, right, flexion and extension) were pooled, whereas multi-segment indices (MSI and MSV) gave single values. Vertebral levels from L2-S1 were analysed in the sagittal plane and from L2-5 in the coronal plane, (given the lack of movement of L5-S1 in this plane). All data were pseudonymised and stored on an encrypted database, with access restricted to the chief investigator, the research assistant and the database manager. Image and statistical analyses were conducted by two independent observers who were blinded to each other's observations. Translation and disc height measures were confined to the sagittal plane, and ICR was excluded due to insufficient segments with rotations above $5^{\circ}$. The study was conducted in accordance with Statistical Methods in Medical Research (SMMR) recommendations [11].

Statistical analysis Data were inspected for distribution and central tendency. Analysis was according to intervertebral level and direction, i.e. left and right from L2-3 to L4-5 (3 levels) and flexion and extension from L2-3 to L5-S1 (4 levels). The association between test and retest and between differences and means was assessed using Kendall's tau. As no significant and/or substantial associations were found, the data were not transformed. Repeatability was assessed using intraclass correlation coefficients ( $\mathrm{ICC}_{2,1}$ - two-way random effects, average measures model) and the minimal detectable change $\left(\mathrm{MDC}_{95}\right)$. To interpret the relevance of the ICC 
'reliability' level, an ICC score of $>0.80$ was considered 'excellent', > 0.60-0.80 'substantial', 0.40-0.60 'moderate' and $<0.40$ 'slight' [44]. This framework is consistent with other reliability studies reporting reliability of spinal posture measurement $[45,46]$.

The distributions of the differences between baseline and follow-up measures for each level and direction for each variable were checked for normality using the Shapiro-Wilk test and the significance of any differences determined. Repeatability coefficients were calculated using the formula below, where $S_{\mathrm{w}}$ is the within-subject standard deviation. The repeatability coefficient estimates the magnitude of the within-subject change that can be expected $95 \%$ of the time and represents the minimum detectable change $\left(\mathrm{MDC}_{95}\right)$ [11].

Table 1 Study populations imaged in each plane

\begin{tabular}{lll}
\hline & Sagittal & Coronal \\
\hline$n$ & 55 & 54 \\
Females & 22 & 21 \\
Age (mean, SD) & $38(13.9)$ & $39(13.6)$ \\
Height (mean, SD) & $1.8(0.1)$ & $1.7(0.1)$ \\
Weight (mean, SD) & $74.7(13.9)$ & $72.6(10.7)$ \\
BMI (mean, SD) & $24.1(2.9)$ & $23.8(2.5)$ \\
\hline
\end{tabular}

Repeatability coefficient $\left(\mathrm{MDC}_{95}\right)=2.77 S_{w}$

\section{Results}

The study population consisted of 43 females and 66 males. Their characteristics and allocations to coronal and sagittal plane investigations are given in Table 1. For those participants who undertook coronal plane investigations, the median effective dose was $0.97 \mathrm{mSv}(1.2 \mathrm{mSv}$ upper third quartile), and for those who undertook sagittal plane investigations, the median effective dose was $0.66 \mathrm{mSv}(0.78 \mathrm{mSv}$ upper third quartile). This is less than and compares favourably to the $1.3 \mathrm{mSv}$ quoted as the typical effective dose expected during a series of X-rays of the lumbar spine for diagnostic procedures [47]. The mean baseline and reference ranges, RMS differences between baseline and follow-up, ICCs $(95 \% \mathrm{CI})$ and $\mathrm{MDC}_{95}$ in the units of the measures and as a percentage of the baseline scores are given in Table 2 for passive recumbent motion and in Table 3 for active weightbearing motion.

In general, reference ranges for IV-RoM and laxity were similar to published control studies that used the same measurement methodology $[22,24,48]$. Their weight-bearing and recumbent values were similar when the same trunk bending range was applied. MSI and MSV, however, had higher

Table 2 Passive recumbent motion: pooled means, RMS differences between baseline and 6-week follow-up, ICCs and MDCs for intervertebral motion parameters in healthy participants

\begin{tabular}{|c|c|c|c|c|c|c|c|}
\hline \multirow{2}{*}{$\begin{array}{l}\text { Direction } \\
\text { Left }\left(40^{\circ}\right)\end{array}$} & \multirow{2}{*}{$\begin{array}{l}\text { Parameter } \\
\text { IV-RoM (degrees) }\end{array}$} & \multirow{2}{*}{$\begin{array}{l}n \\
200\end{array}$} & \multicolumn{2}{|c|}{$\begin{array}{l}\text { Mean baseline ( } \pm 1.96 \mathrm{SD}) \\
\text { RMS (BL-FU) }\end{array}$} & \multirow{2}{*}{$\begin{array}{l}\mathrm{ICC}_{2,1}(95 \% \mathrm{CI}) \\
0.95(0.92-0.96)\end{array}$} & \multirow{2}{*}{$\begin{array}{l}\mathrm{MDC}_{95} \\
3.7\end{array}$} & \multirow{2}{*}{$\begin{array}{c}\text { MDC (\% } \\
\text { baseline) }\end{array}$} \\
\hline & & & $5.37(-0.23$ to 10.97$)$ & 1.33 & & & \\
\hline & Laxity (initial attainment rate) & 188 & $0.19(0.00-0.38)$ & 0.06 & $0.91(0.88-0.93)$ & 0.16 & 85 \\
\hline & Motion sharing inequality (L2-5) & 50 & $0.23(-0.02$ to 0.47$)$ & 0.07 & $0.91(0.84-0.95)$ & 0.21 & 92 \\
\hline & Motion sharing variability (L2-5) & 50 & $0.08(0.00-0.15)$ & 0.04 & $0.37(-0.10$ to 0.64$)$ & 0.12 & 150 \\
\hline \multirow[t]{4}{*}{ Right $\left(40^{\circ}\right)$} & IV-RoM (degrees) & 200 & $5.42(0.00-10.84)$ & 1.18 & $0.95(0.94-0.96)$ & 3.26 & 60 \\
\hline & Laxity (initial attainment rate) & 184 & $0.20(0.00-0.40)$ & 0.06 & $0.91(0.88-0.93)$ & 0.17 & 83 \\
\hline & Motion sharing inequality (L2-5) & 50 & $0.22(-0.05$ to 0.49$)$ & 0.09 & $0.89(0.81-0.94)$ & 0.24 & 110 \\
\hline & Motion sharing variability (L2-5) & 50 & $0.07(0.00-0.14)$ & 0.04 & $0.47(0.08-0.70)$ & 0.12 & 167 \\
\hline \multirow[t]{6}{*}{ Flexion $\left(40^{\circ}\right)$} & IV-RoM (degrees) & 219 & $5.14(1.12-9.16)$ & 1.68 & $0.80(\mathrm{Q} 74-0.84)$ & 4.66 & 91 \\
\hline & Translation (Eqv mm) & 219 & $0.83(-0.14$ to 1.81$)$ & 0.50 & $0.70(0.61-0.77)$ & 1.39 & 167 \\
\hline & Laxity (initial attainment rate) & 213 & $0.16(0.03-0.30)$ & 0.07 & $0.69(0.60-0.77)$ & 0.18 & 111 \\
\hline & Minimum anterior disc height (Eq mm) & 219 & $8.93(4.62-13.24)$ & 1.45 & $0.88(0.84-0.91)$ & 4.02 & 45 \\
\hline & Motion sharing inequality (L2-S1) & 54 & $0.28(0.06-0.50)$ & 0.11 & $0.61(0.34-0.78)$ & 0.31 & 111 \\
\hline & Motion sharing variability (L2-S1) & 54 & $0.08(0.01-0.15)$ & 0.04 & $0.41(0.00-0.66)$ & 0.12 & 158 \\
\hline \multirow[t]{6}{*}{ Extension $\left(40^{\circ}\right)$} & IV-RoM (degrees) & 216 & $5.33(0.68-9.98)$ & 1.87 & $0.80(0.74-0.85)$ & 5.19 & 97 \\
\hline & Translation (Eq mm) & 216 & $-0.80(-2.00$ to 0.40$)$ & 0.60 & $0.64(0.53-0.72)$ & 1.67 & 209 \\
\hline & Laxity (initial attainment rate) & 208 & $0.17(0.03-0.31)$ & 0.07 & $0.71(0.62-0.78)$ & 0.19 & 114 \\
\hline & Maximum anterior disc height (Eq $\mathrm{mm}$ ) & 216 & $12.67(6.39-18.94)$ & 1.90 & $0.89(\mathrm{Q} 86-0.92)$ & 5.27 & 42 \\
\hline & Motion sharing inequality (L2-S1) & 52 & $0.30(0.05-0.55)$ & 0.14 & $0.43(0.020 .67)$ & 0.39 & 131 \\
\hline & Motion sharing variability (L2-S1) & 52 & $0.10(-0.01-0.21)$ & 0.07 & $0.14(-0.05$ to 0.50$)$ & 0.20 & 208 \\
\hline
\end{tabular}


Table 3 Active weight-bearing motion: pooled means, RMS differences between baseline and 6-week follow-up, ICCs and MDCs for intervertebral motion parameters in healthy participants

\begin{tabular}{|c|c|c|c|c|c|c|c|}
\hline Direction & Parameter & $n$ & Mean baseline ( \pm 1.96 SD) & RMS (BL-FU) & $\mathrm{ICC}_{2,1}(95 \% \mathrm{CI})$ & $\mathrm{MDC}_{95}$ & $\begin{array}{l}\text { MDC (\% } \\
\text { baseline) }\end{array}$ \\
\hline \multirow[t]{4}{*}{ Left $\left(40^{\circ}\right)$} & IV-RoM (degrees) & 200 & $4.63(-1.15-10.42)$ & 1.62 & $0.92(0.90-0.94)$ & 4.49 & 97 \\
\hline & Laxity (initial attainment rate) & 170 & $0.17(-0.02$ to 0.35$)$ & 0.08 & $0.80(0.73-0.85)$ & 0.23 & 139 \\
\hline & $\begin{array}{l}\text { Motion sharing inequality } \\
\text { (L2-5) }\end{array}$ & 50 & $0.37(0.02-0.73)$ & 0.18 & $0.69(0.46-0.82)$ & 0.50 & 135 \\
\hline & $\begin{array}{l}\text { Motion sharing variability } \\
\text { (L2-5) }\end{array}$ & 50 & $0.14(0.00-0.28)$ & 0.08 & $0.47(0.07-0.70)$ & 0.22 & 159 \\
\hline \multirow[t]{4}{*}{ Right $\left(40^{\circ}\right)$} & IV-RoM (degrees) & 200 & $4.76(-0.75$ to 10.27$)$ & 1.69 & $0.90(0.87-0.92)$ & 4.68 & 98 \\
\hline & Laxity (initial attainment rate) & 174 & $0.17(-0.02$ to 0.37$)$ & 0.08 & $0.82(0.76-0.87)$ & 0.22 & 125 \\
\hline & $\begin{array}{l}\text { Motion sharing inequality } \\
\text { (L2-5) }\end{array}$ & 50 & $0.31(0.03-0.58)$ & 0.12 & $0.83(0.71-0.91)$ & 0.33 & 108 \\
\hline & $\begin{array}{l}\text { Motion sharing variability } \\
\text { (L2-5) }\end{array}$ & 50 & $0.13(0.02-0.24)$ & 0.07 & $0.40(-0.05$ to 0.66$)$ & 0.20 & 156 \\
\hline \multirow[t]{6}{*}{ Flexion $\left(60^{\circ}\right)$} & IV-RoM (degrees) & 216 & $9.14(0.92-17.37)$ & 3.28 & $0.82(0.76-0.86)$ & 9.10 & 100 \\
\hline & Translation (Eqv mm) & 216 & $1.34(-0.44$ to 3.11$)$ & 0.76 & $0.78(0.71-0.91)$ & 2.10 & 157 \\
\hline & Laxity (initial attainment rate) & 208 & $0.17(-0.07$ to 0.41$)$ & 0.13 & $0.64(0.53-0.73)$ & 0.37 & 219 \\
\hline & $\begin{array}{l}\text { Minimum anterior disc height } \\
(\text { Eq } \mathrm{mm})\end{array}$ & 216 & $8.29(3.32-13.25)$ & 1.58 & $0.89(0.86-0.92)$ & 4.39 & 53 \\
\hline & $\begin{array}{l}\text { Motion sharing inequality } \\
\text { (L2-S1) }\end{array}$ & 52 & $0.39(0.13-0.65)$ & 0.11 & $0.76(0.58-0.86)$ & 0.31 & 78 \\
\hline & $\begin{array}{l}\text { Motion sharing variability } \\
\text { (L2-S1) }\end{array}$ & 52 & $0.17(0.00-0.34)$ & 0.08 & $0.65(0.39-0.80)$ & 0.23 & 135 \\
\hline \multirow[t]{6}{*}{ Extension $\left(20^{\circ}\right)$} & IV-RoM (degrees) & 217 & $3.17(-3.17$ to 9.51$)$ & 2.01 & $0.91(0.88-0.93)$ & 5.53 & 176 \\
\hline & Translation (Eq mm) & 218 & $-0.39(-1.16$ to 0.37$)$ & 0.4 & $0.55(0.42-0.66)$ & 1.12 & 283 \\
\hline & Laxity (initial attainment rate) & 171 & $0.16(-0.08$ to 0.4$)$ & 0.12 & $0.73(0.64-0.80)$ & 0.32 & 205 \\
\hline & $\begin{array}{l}\text { Maximum anterior disc height } \\
(\text { Eq mm) }\end{array}$ & 213 & $12.44(6.76-18.11)$ & 2.03 & $0.85(0.81-0.89)$ & 5.64 & 45 \\
\hline & $\begin{array}{l}\text { Motion sharing inequality } \\
\text { (L2-S1) }\end{array}$ & 53 & $0.49(0.11-0.86)$ & 0.21 & $0.57(0.25-0.76)$ & 0.59 & 122 \\
\hline & $\begin{array}{l}\text { Motion sharing variability } \\
\text { (L2-S1) }\end{array}$ & 53 & $0.09(0.00-0.18)$ & 0.13 & $0.41(-0.02$ to 0.66$)$ & 0.37 & 408 \\
\hline
\end{tabular}

values during weight bearing than recumbent motion for all directions.

Reliability Reliability was substantial to excellent for repeated measurements of IV-RoM, laxity, flexion translation and disc height during recumbent passive (ICC 0.69-0.96) and active weight-bearing motion (ICC 0.64-0.92), except that translation was only moderate for weight-bearing extension translation (ICC 0.55). MSI was moderate to excellent for both positions (ICC 0.43-0.91), and MSV was moderate to substantial for weight-bearing motion (ICC 0.40-0.65), but poor to moderate for recumbent motion (ICC 0.14-0.47).

Measurement error Measurement errors $\left(\mathrm{MDC}_{95}\right)$ for all variables were high, ranging from $42 \%$ of baseline for anterior disc height in passive recumbent extension to $408 \%$ for weight-bearing extension MSV, suggesting that degrees of change that would be of interest may not be detected in these ranges (Tables 2, 3). Measures of restraint (IV-RoM and laxity) tended to have lower measurement errors in recumbent passive than active weight-bearing motion. However, of all the measures, anterior disc height had the smallest measurement errors, ranging from $45 \%$ of baseline in recumbent extension to $53 \%$ in weight-bearing flexion. The measurement error for translation was unacceptably high for both weight-bearing (157-283\%) and recumbent (111-209\%) tests, possibly reflecting their small baseline values in healthy controls. For MSV, weight-bearing measurement error ranged from 135 to $408 \%$ and recumbent from 150 to $208 \%$, while MSI was $78-135 \%$ for weight bearing and $91-131 \%$ for recumbent. Measurement error for disc height, on the other hand, ranged from $42 \%$ for passive extension to $53 \%$ for weight-bearing flexion. 


\section{Discussion}

This is the first appearance of intrasubject repeatability studies of in vivo continuous intervertebral motion parameters using controlled motion protocols, and the first time to our knowledge that spine biomechanical measurement error has been calculated over a clinically relevant outcome interval. The results suggest that, irrespective of baseline measurement values, follow-up data would not necessarily be useful as biomechanical outcomes for all measures: This is simply because there is poor repeatability of some variables. On the other hand, the acceptable levels of reliability bode well for their use for distinguishing between low back pain patients in relation to biomechanical change [27].

A summary of the magnitudes of reliability and measurement error for all variables is given in Table 4. This shows that for outcome studies that employ QF, the best overall intrasubject reliability and agreement over a 6-week intervention period are the measurement of disc height and IV-RoM and the worst for the measurement of MSV. The measurement of laxity, MSI and translation has acceptable reliability, but not agreement. The implications of this for outcome studies is that for the time being, disc height and IV-RoM are the only variables that could be considered for randomised trials of interventions that might target these as outcomes. With the exception of MSV, the other variables (laxity, MSI and translation) could be considered for investigation as baseline moderators or perhaps correlates or mediators of patient-reported outcomes.

Limitations Results for individual-level vertebral data were not calculated in this study as the aim was to address repeatability and the differences between baseline and follow-up measures. In addition, some measures, such as translation, had low values in healthy controls and their changes across time, although small, would be high compared to the baseline itself, giving high percentages but low errors (e.g. 1-2 equivalent $\mathrm{mm}$ for translation) which could be quite acceptable in patients with high baseline values. Therefore, patients with high translation or laxity values may have values that are expected to be reduced greatly by an intervention (such as spinal fusion) again, making high measurement error more tolerable. For example, the $\mathrm{MDC}_{95}$ for recumbent laxity of between 0.16 and 0.19 is a difference that would be likely to be detected as the upper reference levels are in the region of 0.40 .

The variables evaluated in this study may have greater clinical utility as observational measures rather than specific outcomes to detect change over time, especially for recumbent testing, where there was excellent reliability for a number of measures including: IV-ROM, laxity, disc height and MSI. On the other hand, recumbent IV-RoM and laxity produced the smallest measurement errors, ranging from 55 to $97 \%$, suggesting that these measures of restraint show some promise for longitudinal testing of change over time. Evaluation of recumbent motion enables spinal motion analysis to be conducted without the influence of muscular control and tend to be much better tolerated by individuals who are in pain. Subsequently, variables measured in this position may be biomarkers for LBP [5, 42].

Variables tested during weight bearing generally demonstrated slightly lower reliability scores and higher errors over time compared to recumbent testing. Spinal movement during weight-bearing studies involves active control; thus, muscle activation is likely to play a role in the magnitude of such variables. Future work could therefore include evaluation of the active components of spinal movement, for example, muscle activity using electromyography and muscle oxidation and perfusion to understand potential mechanisms underpinning motor control and muscle metabolism in both the symptomatic and asymptomatic spines during dynamic movement.

Measures of proportional motion inequality (MSI) and variability (MSV) of lumbar motion using QF have shown promise in differentiating between healthy and CLBP populations [22, 42]. MSI has been shown to be significantly
Table 4 Summary table of magnitudes of reliability ${ }^{\epsilon}$ and measurement error for all variables

\begin{tabular}{|c|c|c|c|c|}
\hline & \multicolumn{2}{|l|}{ Reliability $\left(\mathrm{ICC}_{2,1}\right)$} & \multicolumn{2}{|c|}{ Measurement error $\left(\mathrm{MDC}_{95}\right)$} \\
\hline & Weight bearing & Recumbent & Weight bearing & Recumbent \\
\hline IV-RoM & Excellent & Excellent & $2(\mathrm{~L}+\mathrm{R}+\mathrm{Flx})$ & 2 (all) \\
\hline Laxity & Moderate/excellent & Substantial/excellent & $4(\mathrm{~L}+\mathrm{R}+\mathrm{Flx})$ & 3 (all) \\
\hline MSI & Substantial/excellent & Substantial/excellent & 3 & 3 \\
\hline MSV & Moderate & Slight/moderate & $3(\mathrm{~L}+\mathrm{R}+\mathrm{Flx})$ & $3(\mathrm{~L}+\mathrm{R}+\mathrm{Flx})$ \\
\hline *Translation (flexion) & Substantial & Substantial & 3 & 3 \\
\hline *Disc height (flexion) & Excellent & Excellent & 2 & 1 \\
\hline
\end{tabular}

Legend for $\mathrm{MDC}_{95}:<50 \%=1,50-100 \%=2,100-200 \%=3,>200 \%=4$

${ }^{\epsilon}$ Landis and Koch [44]

*Magnitudes were only reported for flexion owing to the small range of extension $\left(20^{\circ}\right)$ in weight bearing 
greater and, notably, correlated with composite disc degeneration (CDD) in CLBP during recumbent flexion [5]. This suggests greater inequality of motion sharing in NSLBP individuals and intimates a link between in vivo biomechanics of the disc and pain. MSI's reliability in the current study, as represented by intraclass correlations, was generally acceptable for both weight-bearing and recumbent measures; thus, MSI may be a useful variable of interest for future clinical QF studies.

Although QF protocols were associated with acceptable intrasubject repeatability for some parameters, the poor intrasubject results observed for MSV may be hypothesised to be due to individual changes in the behavioural performance of spinal motion rather than measurement error, although variability of movement is fundamental to motor learning and control, especially in the study of healthy movement and posture [49]. In order to repeatedly achieve a task consistently, variability is required in the motor constituents, to ensure that the individual can respond to altered task demands without performance being compromised [49]. Thus, one could hypothesise that healthy individuals demonstrate unique movement behaviours and may have a range of potential movement patterns available which may explain the high error values obtained for MSV.

Further work The results of this study support previous work that has demonstrated the intra- and interobserver repeatability of these measures [24-26, 48, 50], However, this still needs to be determined for MSI and MSV. We also suggest that the present methodology should be repeated in a stable CLBP cohort, where baseline parameters may be different.

\section{Conclusion}

Of the six measurement parameters considered, disc height and IV-RoM were the only variables that could currently be considered for use in randomised trials of interventions that employ these as outcome measures. However, laxity, MSI and translation could be considered as candidates for potential moderators, correlates or mediators of patient-reported outcomes.

Acknowledgements $\mathrm{AB}$ received a project grant from the European Chiropractors Union Research Fund (ECURF), and RH received a Seedcorn Bursary from the Cardiff Institute of Tissue Engineering and Repair (CITER).

\section{Compliance with ethical standards}

Conflict of interest The authors declare that they have no conflict of interest.
Open Access This article is distributed under the terms of the Creative Commons Attribution 4.0 International License (http://creativeco mmons.org/licenses/by/4.0/), which permits unrestricted use, distribution, and reproduction in any medium, provided you give appropriate credit to the original author(s) and the source, provide a link to the Creative Commons license, and indicate if changes were made.

\section{References}

1. Hoy D, March L, Brooks P, Blyth F, Woolf A, Bain C, Williams G, Smith E, Vos T, Barendregt J, Murray C, Burstein R, Buchbinder R (2014) The global burden of low back pain: estimates from the Global Burden of Disease 2010 study. Ann Rheum Dis 73:968-974

2. Deyo RA, Rainville J, Kent DL (1992) What can the history and physical examination tell us about low back pain? JAMA 268:760-765

3. Cheng JS, Carr CB, Wong C, Sharma A, Mahfouz MR, Komistek RD (2013) Altered spinal motion in low back pain associated with lumbar strain and spondylosis. Evid Based Spine Care J 4:6-12. https://doi.org/10.1055/s-0033-1341640

4. Teyhen DS, Flynn Tw, Childs JD, Abraham LD (2007) Arthrokinematics in a subgroup of patients likely to benefit from a lumbar stabilization exercise program. Phys Ther 87:313-325

5. Breen A, Breen A (2018) Uneven intervertebral motion sharing is related to disc degeneration and is greater in patients with chronic, non-specific low back pain: an in vivo, cross-sectional cohort comparison of intervertebral dynamics using quantitative fluoroscopy. Eur Spine J 27:145-153. https://doi.org/10.1007/ s00586-017-5155-y

6. Wang Q, Liu J, Shi Y, Chen Y, Yu H, Ma J, Ren W, Yang H, Wang H, Xiang L (2016) Short-term effects of a dynamic neutralization system (Dynesys) for multi-segmental lumbar disc herniation. Eur Spine J 25:1409-1416

7. Beastall J, Karadimas E, Siddiqui M, Nicol M, Hughes J, Smith F, Wardlaw D (2007) The Dynesys lumbar spinal stabilization system: a preliminary report on positional magnetic resonance imaging findings. Spine 32:685-690

8. Powers CM, Kulig K, Harrison J, Bergman G (2003) Segmental mobility of the lumbar spine during a posterior to anterior mobilization: assessment using dynamic MRI. Clin Biomech 18:80-83

9. Volkow ND, McLellan AR (2016) Opioid abuse in chronic pain-misconceptions and mitigation strategies. N Engl J Med 374:1253-1263

10. Kessler LG, Barnhart HX, Buckler AJ, Choudhury KR, Kondratovich MV, Toledano AY, Guimaraes AR, Filice R, Zhang Z, Sullivan DC, Group aQTW (2015) The emerging science of quantitative imaging biomarkers terminology and definitions for scientific studies and regulatory submissions. Stat Methods Med Res 24:9-26

11. Bland JM, Altman DG (1996) Statistics notes: measurement error. Br Med J 313:744

12. Bauer CM, Heimgartner M, Rast FM, Ernst MJ, Oetiker S, Kool J (2016) Reliability of lumbar movement dysfunction tests for chronic low back pain patients. Man Ther 24:81-84

13. Goel VK, Goyal S, Clark C, Nishiyama K, Nye T (1985) Kinematics of the whole lumbar spine: effect of discectomy. Spine 10:543-564

14. Schultz AB, Warwick DN, Berkson MH, Nachemson AL (1979) Mechanical properties of human lumbar spine motion segments-Part 1: responses inflexion, extension, lateral bending and torsion. J Biomech Eng 101:46-52 
15. Tencer AF, Ahmed AM, Burke DL (1982) Some static mechanical properties of the lumbar intervertebral joint, intact and injured. J Biomech Eng 104:193-201

16. Bassini T, Stucovitz E, Qian Z, Briguglio M, Galbusera F (2017) Validation of the AnyBody full body musculoskeletal model in computing lumbar spine loads at L4L5 level. J Biomech 58:89-96

17. Shirazi-Adl A, Ahmed AM, Shrivastava SC (1986) A finite element study of a lumbar motion segment subjected to pure sagittal plane moments. J Biomech 19:331-350

18. Oxland TR (2016) Fundamental biomechanics of the spine-what we have learned in the past 25 years and future directions. J Biomech 49:817-832

19. Jones AC, Wilcox RK (2008) Finite element analysis of the spine: towards a framework of verification, validation and sensitivity analysis. Med Eng Phys 30:1287-1304

20. Iguchi T, Kanemura A, Kasahara K, Sato K, Kurihara A, Yoshiya S, Nishida K, Miyamoto H, Doita M (2004) Lumbar instability and clinical symptoms. Which is the more critical factor for symptoms: sagittal translation or segment angulation? J Spinal Disord Tech 17:284-290

21. Breen AC, Teyhen DS, Mellor FE, Breen AC, Wong K, Deitz A (2012) Measurement of inter-vertebral motion using quantitative fluoroscopy: report of an international forum and proposal for use in the assessment of degenerative disc disease in the lumbar spine. Adv Orthop 2012:1-10. https://doi.org/10.1155/2012/802350

22. Mellor FE, Thomas P, Thompson P, Breen AC (2014) Proportional lumbar spine inter-vertebral motion patterns: a comparison of patients with chronic non-specific low back pain and healthy controls. Eur Spine J 23:2059-2067. https://doi.org/10.1007/ s00586-014-3273-3

23. Amevo B, Aprill C, Bogduk N (1992) Abnormal instantaneous axes of rotation in patients with neck pain. Spine 17:748-756

24. du Rose A, Breen A (2016) Relationships between lumbar intervertebral motion and lordosis in healthy adult males: a cross sectional cohort study. BMC Musculoskeletal Disord 17:121

25. Breen A (2011) Quantitative fluoroscopy and the mechanics of the lumbar spine. Open University

26. Breen A, Breen A (2016) Accuracy and repeatability of quantitative fluoroscopy for the measurement of sagittal plane translation and instantaneous axis of rotation in the lumbar spine. Med Eng Phys 38:607-614

27. de Vet HCW, Terwee CB, Knol DL, Bouter LM (2006) When to use agreement versus reliability measures. J Clin Epidemiol 59:1033-1039

28. Shrout PE, Fleiss JL (1979) Intraclass correlations: uses in assessing rater reliability. Psychol Bull 86:420-428

29. Posner IRA, White AA, Edward WT, Hayes WC (1982) A biomechanical analysis of the clinical stability of the lumbar and lumbosacral spine. Spine 7:374-389

30. Van Herp G, Rowe P, Salter P, Paul JP (2000) Three-dimensional lumbar spinal kinematics: a study of range of movement in 100 healthy subjects aged 20 to $60+$ years. Rheumatology 39:1337-1340

31. Dvorak J, Panjabi MM, Chang DG, Theiler R, Grob D (1991) Functional radiographic diagnosis of the lumbar spine. Flexionextension and lateral bending. Spine 16:562-571

32. Branney $\mathbf{J}$ (2014) An observational study of changes in cervical inter-vertebral motion and the relationship with patient-reported outcomes in patients undergoing spinal manipulative therapy for neck pain. Bournemouth University, Poole

33. du Rose A, Breen A (2016) Relationships between lumbar intervertebral motion and lordosis in healthy adult males: a cross sectional cohort study. BMC Musculoskeletal Disord 17:121. https ://doi.org/10.1186/s12891-016-0975-1
34. Frobin W, Brinckmann P, Biggemann M, Tillotson M, Burton K (1997) Precision measurement of disc height, vertebral height and sagittal plane displacement from lateral radiographic views of the lumbar spine. Clin Biomech 12:S22-S30

35. Breen A, Breen A (2016) Accuracy and repeatability of quantitative fluoroscopy for the measurement of sagittal plane translation and finite centre of rotation in the lumbar spine. Med Eng Phys 38:607-614. https://doi.org/10.1016/j.medengphy.2016.03.009

36. Mellor F, Muggleton JM, Bagust J, Mason W, Thomas PW, Breen AC (2009) Mid-lumbar lateral flexion stability measured in healthy volunteers by in vivo fluoroscopy. Spine 34:E811-E817. https://doi.org/10.1097/brs.0b013e3181b1feba

37. Breen AC, Dupac M, Osborne N (2015) Attainment rate as a surrogate indicator of the intervertebral neutral zone length in lateral bending: an in vitro proof of concept study. Chiropr Man Therap 23:28. https://doi.org/10.1186/s12998-015-0073-8

38. Panjabi MM (2003) Clinical spinal instability and low back pain. J Electromyogr Kinesiol 13:371-379

39. Breen AC, Dupac M, Osborne N (2015) Attainment rate as a surrogate indicator of the intervertebral neutral zone length in lateral bending: an in vitro proof of concept study. Chiropr Man Therap 23:28

40. Breen AC, Teyhen DS, Mellor FE, Breen AC, Wong KWN, Deitz A (2012) Measurement of intervertebral motion using quantitative fluoroscopy: report of an international forum and proposal for use in the assessment of degenerative disc disease in the lumbar spine. Adv Orthop 2012:802350. https://doi.org/10.1155/2012/802350

41. Johnsen LG, Brinckmann P, Hellum C, Rossvoll I, Leivseth G (2013) Segmental mobility, disc height and patient-reported outcomes after surgery for degenerative disc disease. A prospective randomised trial comparing disc replacement and multidisciplinary rehabilitation. Bone Joint J 95-B:81-89

42. Breen A, Mellor F, Breen A (2018) Aberrant intervertebral motion in patients with treatment-resistant nonspecific low back pain: a retropective cohort study and control comparison. Eur Spine J. https://doi.org/10.1007/s00586-018-5666-1

43. Bogduk N, Amevo B, Pearcy M (1995) A biological basis for instantaneous centres of rotation of the vertebral column. Proc Inst Mech Eng 209:177-183

44. Landis JR, Koch GG (1977) The measurement of observer agreement for categorical data. Biometrics 33:159-174

45. Sheeran L, Sparkes V, Busse M, van Deursen R (2010) Preliminary study: reliability of the spinal wheel. A novel device to measure spinal postures applied to sitting and standing. Eur Spine J 19:995-1003

46. O’Sullivan K, Galeotti L, Dankaerts W, O'Sullivan L, O'Sullivan $P$ (2011) The between-day and inter-rater reliability of a novel wireless system to analyse lumbar spine posture. Ergonomics 54:82-90. https://doi.org/10.1080/00140139.2010.535020

47. England PH (2008) Patient dose information: guidance. In. Gov. uk, London

48. Mellor F, Muggleton JM, Bagust J, Mason W, Thomas PW, Breen AC (2009) Mid-lumbar lateral flexion stability measured in healthy volunteers by in vivo fluoroscopy. Spine 34:E811-E817

49. Moseley GLH, Hodges PW (2006) Reduced variability of postural strategy prevents normalisation of motor changes induced by back pain: a risk factor for chronic trouble? Behav Neurosci 120:474-476

50. Breen A, Muggleton J, Mellor F (2006) An objective spinal motion imaging assessment (OSMIA): reliability, accuracy and exposure data. BMC Musculoskeletal Disord 7:1-10 


\section{Affiliations}

\section{Alexander Breen ${ }^{1} \cdot$ Rebecca Hemming ${ }^{2} \cdot$ Fiona Mellor $^{1} \cdot$ Alan Breen $^{3}$ (ID}

1 Centre for Biomechanics Research, AECC University College, Parkwood Road, Bournemouth, Dorset BH5 2DF, UK

2 Arthritis Research UK Biomechanics and Bioengineering Centre, School of Healthcare Sciences, Cardiff University, Cardiff, UK
3 Faculty of Science and Technology, Bournemouth University, Bournemouth, UK 